\title{
PLANNING AND SCHEDULING IN AN FMS*
}

\author{
E. Aanen, G.J. Gaalman \\ Department of Mechanical Engineering, University of Twente, Enschede (The Netherlands) \\ and W.M. Nawijn \\ Department of Applied Mathematics, University of Twente, Enschede (The Netherlands)
}

\section{ABSTRACT}

The Dutch institute of applied physics of TNO and the Institute of Metals TNO (Apeldoorn) have installed a flexible manufacturing system (FMS). The main components of this FMS are a turret lathe, a machining centre and a transport system. One of the objectives of this system is to produce a wide variety of parts in small batches. Several interesting (short term) planning and scheduling problems arise from this objective. Among others, (1) which orders should be processed in a certain period, and (2) in what

\section{INTRODUCTION}

During the last decade there has been a shift from productivity towards flexibility in manufacturing. This is seen by the number of flexible manufacturing systems (FMSs) that have been installed and the number that will be installed in the near future (see, for example [1]).

The advantages of FMS's are, among others:

- the possibility to produce economically in small batches, because of the relatively short set-up times;

- the possibility to produce a rather wide range of part types;

\footnotetext{
*Presented at the 5th International Working Seminar on Production Economics, Igls, Austria, February 22-26, 1988.
}

sequence should the selected orders be processed. In solving these problems, one has to take into account due dates, process plans (routing), capacities of the machines and the tool magazines, tool and jaw changing times, limited fixturing capacity, fixturing and clamping times and the limited number of operators and transport devices. The paper describes the typical characteristics of the FMS with respect to the planning and scheduling problems. A way to solve these problems is presented.

- short lead times, low in-process inventories, high machine utilization; and

- high quality.

The range of different part types to be manufactured in industry may vary widely. In the "average" Dutch job shop many part types, between 50 and 200, are machined in small batches, 1-10 parts per batch. For this typical situation it is expected that FMSs should be able to manufacture 50-200 part types in small batches. The Dutch institute TNO has installed a small FMS in order to get experience with flexible manufacturing for this situation. The FMS consists of one turret lathe and one machining centre. The integration of these and other components of the FMS is realized by means of complicated software, the so called supervisory control system (SCS). This SCS, 
developed by TNO, is a set of software modules which perform functions as: accepting orders from outside; giving signals to and accepting signals from machines and transportsystem; storing, retrieval and reporting of relevant data. Part of this SCS is the production planning and scheduling function. As one of the partners in the TNO-FMS project we have developed a planning/scheduling strategy.

In the second section we describe the main characteristics of the FMS. In the third section the production planning/scheduling problem is introduced. In the fourth section a way to handle this difficult problem is discussed. The next two sections ( 5 and 6 ) describe the solution approach in more detail.

\section{THE SYSTEM CONFIGURATION}

In Fig. 1 the FMS which is being built by TNO is represented schematically. The FMS consists of the following elements:

(1) One machining centre (MC) with a local tool magazine (LTM) of 48 positions and a local pallet magazine (LPM) consisting of one input and one output buffer in front of the machine. Also in front of the machine is a pallet magazine ('regal system'), which can contain up to 15 pallets. Two places of this magazine can be used for (un )clamping activities $(\mathrm{L} / \mathrm{U})$. A crane can auromatically move pallets from this magazine to the buffers and vice versa;

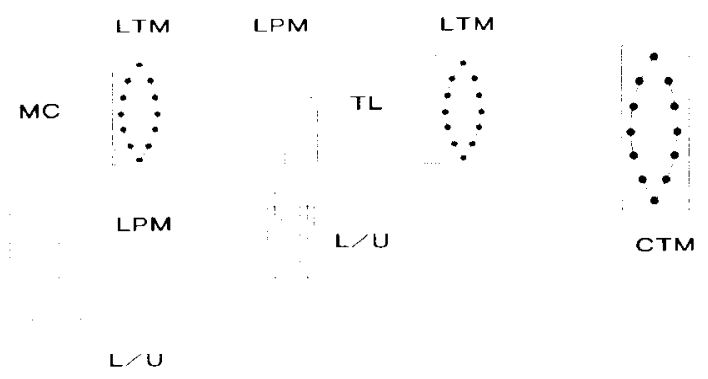

CPM

Fig. 1. The FMS of TNO.
(2) One turret lathe (LT) with a local tool magazine (LTM) of 96 positions. In this LTM not only cutting tools can be placed but also a number of jaws (for rotational parts with different diameters different jaws may be needed). Transport of jaws between the LTM and the spindle is executed by means of a gantry arm. This gantry arm also executes the transport of tools to the turret. The turret has 12 tool positions. Again there is a local part magazine and one load/unload station. A second gantry arm coupled to the first transports parts between the local part magazine and the spindle. Contrary to the MC pallets are internal to the machine;

(3) The planned transport system of the system will either consist of two automated guided vehicles (AGVs) or a conveyor system. For the time being it will be assumed that transport will be done manually by operators. The transport system is not given in Fig. 1;

(4) A central part magazine (CPM). In this CPM raw material, partially manufactured parts and finished products can be stored;

(5) A central tool magazine (CTM), for storing tools that cannot be placed in the LTMs of the machines, because of limited capacity or other reasons. The transport and the exchange of cutting tools must be done by an operator. This FMS has been designed for a product variety of about 70 types.

\section{PROBLEM DESCRIPTION}

Having described the main characteristics of the FMS, we now give a description of the planning/scheduling aspect of the system. In general the machining operations on jobs in the FMS form only one out of several production phases. So the planning of the jobs for the system should be constrained by a higher level production control system (PCS). This will be effectuated by means of an (internal) due date and an availability date for every order to be processed in the FMS. The PCS issues an order list for the FMS, which contains all released or- 
ders with their time window, characterized by an availability date and a due date. Orders are added to the list on a daily (c.q. shift) basis. Each order consists of one or more identical parts with a given process plan. The latter contains the routing over the two machines and the processing times and tools needed. There are two types of activities to be planned and scheduled: machining activities and operator activities.

Essential operator activities are:

- pallet fixturing (attaching or removal fixtures);

- part and tool transport (to and from machines or central magazines );

- part(s) (un)clamping (attaching or removal of a part from a fixtured pallet); and

- tool (un ) loading at the machines.

It will be clear that when scheduling the machining and operator activities the interdependencies should be taken into account. Moreover, it should be noted that especially at the lathe sequence dependent change-over times occur, depending on the tools needed for subsequent parts. These change-over times include transport times of tools (jaws) between the local tool magazine and the turret (spindle).

Given the order list, the planning/scheduling problem which has to be solved can be divided into three subproblems:

\section{(1) On which days should an order be processcd.}

(2) The assignment of resources to activities, such as operators to fixturing or transport activities; when clamping a part an operator and a fixtured pallet are needed.

(3) Scheduling the activities, leading to loading tables for each resource.

In view of the dynamic order release to the order list, it is decided that activities will be scheduled day by day. The order list will typically have a time horizon of about 10 days, containing only orders with due dates within this time horizon.

The above problems have to be solved sub- ject to the constraints imposed by the system configuration and the higher planning level. The main constraints are:

- time window constraints from the order list;

- technological constraints, either induced by the process plans or by the pallet/part fixturing and clamping possibilities, and capacity constraints with respect to tool and pallet magazines; and

- limited number of operators and machines. In planning and scheduling one strives for one or more objectives to be satisfied. In our case the main objective is to satisfy the imposed due dates of the orders. It may, however, not always be possible to meet all due dates, give the available hours per day, in particular if the due dates are too tight. Therefore we will use the relaxed objective of minimizing the total number of late orders within the time horizon. $\Lambda$ part from this objective we want the system to be used as efficiently as possible, in view of the high capital investments. This means that our secondary objective is to minimize the total change-over and idle times of the machines within the time horizon.

\section{SOLUTION APPROACH}

The planning/scheduling problem described before is rather complex and very difficult to solve, because:

(1) the order list does not contain all orders to be produced during the planning horizon, since each new day additional orders are released to the FMS;

(2) there are conflicting objectives, i.e. minimizing the number of late orders versus minimizing change-over and idle times of the machines;

(3) the high number of activities and their interrelations (due to the different number of part types, tool types and fixtures ); and

(4) there are several uncertainties to cope with, such as tool breakage, machine disturbances, variations in human tasks.

For this reason we "simplify" and "decom- 


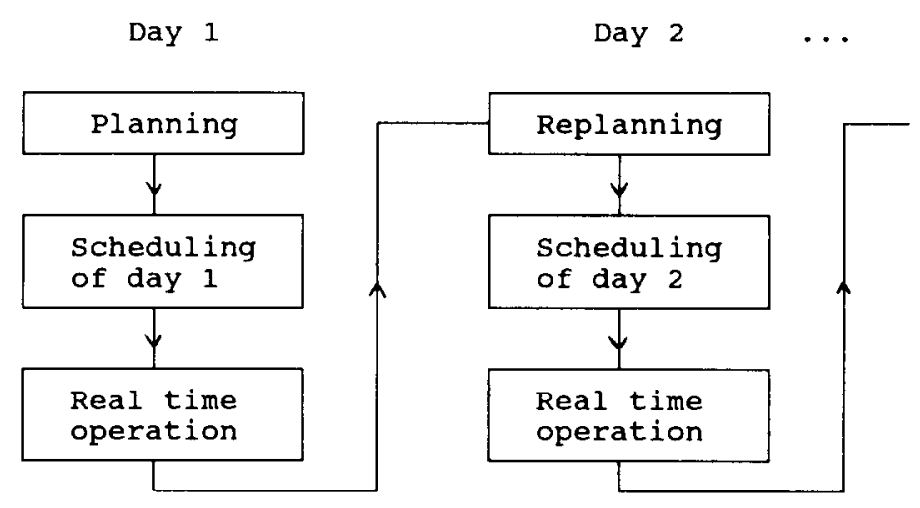

Fig. 2. Planning and scheduling strategy.

pose" the problem. The following simplifications are made:

(a) The number of pallets is unlimited;

(b) Only one part is clamped on a pallet;

(c) Overtime is not possible. There is one shift of 8 hours. During this shift the number of operators is constant;

(d) The durations of all activities, including human activities, are constant;

(e) There are no tool breakages and machine disturbances.

Despite of all these simplifications the problem remains too complex to solve in one step. Therefore we decompose the problem hierarchically as usually done, in a planning problem and a scheduling problem (see, for example $[2,3]$ ). First the planning problem is solved and the resulting output forms the input for the scheduling problem. Generally there is no feedback from scheduling to planning. When the output of the scheduling level is unsatisfactory a feedback to the planning level is recommendable, however, at the costs of additional computations.

At the planning level the time horizon (of about 10 days) is divided in periods of one day. For each day machining activities, to be performed, are assigned. The resulting list is called a day list. It is possible that an individual part is machined over two or more days. This means that the associating machining activities of this part are divided over two or more day lists.
The list of activities for the first day is input to the scheduling level. These machining activities together with the corresponding operator activities are then scheduled.

One may wonder why at the planning level several day lists are generated instead of only the list for the first day. The reasons are:

(1) when only the first day is considered it might be possible that an attractive day list is formed, however, at the expense of suitable future day lists;

(2) it gives a rough idea of future activities to be executed on a certain day, so that necessary preparations can be done; and

(3) it indicates which "future" orders cannot be manufactured within the due dates, so a signal to the PCS can be given in time.

Future day lists are not scheduled yet, because future releases might substantially disturb such schedules.

The planning and scheduling strategy in relation with real time operation is presented in Figure 2.

\section{PLANNING}

Although we have decomposed the planning/scheduling problem into two separate problems, we have not formulated the objective function for each problem. These objectives should be in correspondence with the overall objectives. Only after the scheduling 
problem is solved one can evaluate how well these objectives are satisfied.

The solution at the planning level (that is to say, the generation of day lists) determines to a high degree the conditions at the scheduling level. We mention two possible types of day lists which make a good schedule difficult:

(1) Day lists for which the capacity of at least one of the machines is underutilized. This will result in idle time at the scheduling level.

(2) Day lists where the machining activities use a large number of tools. This may induce high change-over times on the turret lathe. In addition, many tool loading and unloading activities may be necessary. By this, the utilization of operators, that perform these activities, may become temporarily so high, that delays and machine idle times result.

Generally, we expect at the planning level to bc able to form day list without significant under utilization of the machines, regardless of the solution method used. So we concentrate on the prevention of the second type of day lists. This will hopefully be realized by introducing the following objective: minimize the total number of tools needed for the day lists over the planning horizon. This objective is used in addition to the primary objective: minimize the total number of late orders within the planning horizon. The heuristic in forming day lists in correspondence with the objectives consists of two steps: Phase I and Phase II.

\section{Phase I:}

According to the tools needed, clusters of (machining) activities are formed. The time window restrictions are disregarded at this level. The clusters have the following properties:

- the activities within a cluster have a high overlap in tools needed;

- each activity belongs to only one cluster;

- the tool overlap between clusters is small. An interactive clustering technique due to King [4] is used. By forming those clusters we hope that the objective regarding the number of tools needed can be satisfied.

\section{Phase II:}

Assign the activities of each cluster to the smallest number of days possible, taking into account the time window constraints, technological ordering constraints, etc. Here allowance is made for the urgency of the activities (orders), so we hope that the primary objective of minimizing the number of late orders can be satisfied.

In the heuristic we use time windows for (machining) activities. These windows are in principal equal to the windows of the corresponding orders. The output of the first phase is a set of clusters; each cluster contains a set of activities. In order to assign the activities to days we first select the most attractive days at which clusters could be machined. For each cluster $i, i=1, \ldots, N$, we calculate $z_{i c}$ as the number of activities that can be machined on day $c, c=1, \ldots, H$ ( $H=$ the planning horizon $)$. We define $z_{i}$ as $\left(z_{i 1}, z_{i 2}, \ldots, z_{i H}\right)$. Then the elements of $z_{i}$ are ranked in decreasing order. From this a row of decreasing preference days is generated for cluster $i$. We call this row the cluster preference row $r_{i}$. Regrettably an activity within cluster $i$ cannot always be assigned to one of the most preferable days due to the restrictions given by its time window. Therefore we subsequently determine a preference row for each activity which is based on the corresponding cluster preference row $r_{i}$. Starting with the first preference day of the cluster preference row we successively move each day that does not fall within the time window to the end of the row. This results in a row of preference days for which the first entries correspond to the days within the window.

All these rows together form the preference matrix $\boldsymbol{P}$. We will illustrate the determination of the activity preference row by means of a small example. Consider an activity $j$ which 
belongs to a certain cluster $i$. The time window of $j$ is $\langle 1,3\rangle$. The current planning day $c$ is 1 and the horizon $H$ is 5 days. The row $z_{i}$ is assumed to be $(3,12,8,5,1)$. Hence, the number of activities belonging to cluster $i$ which can be machined on day 1 (with respect to their time windows) is 3 , on day 2 this number is 12, etc. From this row $z_{i}$ we derive the preference days for activity $j$. First, the elements in the row $z_{i}$ are ranked in decreasing order, which results in the row $(12,8,5,3,1)$. Now the cluster preference row $r_{i}$ simply constitutes of the corresponding day numbers of this ranked row. Hence, $r_{i}=(2,3,4,1,5)$. The days of this row which fall outside the activity's window are days 4 and 5; therefore these days are moved in unchanged order to the end of the cluster preference row. This linally results in the activity preference row $p_{j}=(2,3,1,4,5)$. The matrix $\boldsymbol{P}$ is input to the algorith $m$ which finally assigns the activities to day lists.

Refore describing this algorithm we introduce the following notation:

$m$ machine number ( $1=$ turret lathe, $2=$ machining centre)

C. current planning day

$D_{c}$. the day list of activities for day $c$

$S_{c m}$ a list of unplanned activities at day $c$ for machine $m$ with availability date $\leqslant c$

$i$ an activity number in $S_{c m}$

$n_{j} \quad$ the number of unplanned successor activitics of activity $j$ plus one

$a_{j} \quad$ the availability date of activity $j$ (equal to the availability date of the order to which activity $j$ belongs )

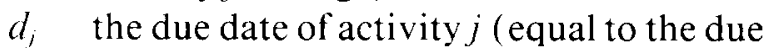
date of the order to which activity $j$ belongs )

$k$ preference number

$\boldsymbol{P} \quad$ preference matrix, element $P_{j k}$ denoted the $k$-th preferable day of activity $j$

\section{Assignment algorithm:}

Step 0: Let the content of the daylists be zero: $D_{1}=D_{2}=\ldots=D_{H}=0$.
Step 1: Set the current planning day $c=1$.

Step 2: Calculate $\boldsymbol{P}$.

Step 3: Set the machine number $m=1$.

Step 4: Set the preference number $k=1$.

Step 5: Create $S_{c m}$. Determine for each activity $j \in S_{c m}$ the urgency function $f_{j}$ : $f_{i}-\left(d_{j}-a_{j}\right) / n_{j}$

Sort $S_{c m}$ in increasing order with respect to $f_{j}$. Let $j=1$.

Step 6: Test whether $p_{j k}$ equals $c$ and whether the technological predecessor of activity $j$ has already been assigned and check if the available hours per day are still sufficient. If this test is positive, add $j$ to day list $D_{c}$.

Step 7: If $j<\left|S_{c m}\right|$ then let $j=j+1$ and go to step 6.

Step 8: If there is still time available for the day and $k<H+1-c$ then let $k=k+1$ and go to step 5 .

Step 9: If $m<2$, take $m=2$ and return to step 4.

Step 10: If $c<H$ then let $c=c+1$, update the time windows and the preference matrix $\boldsymbol{P}$ and go to step 3 .

Step 11: Stop.

In step 10 we update the time windows. That is to say we let the availability time of activity $j$ be equal to the minimum of $a_{j}$ and $c$. We also update $\boldsymbol{P}$ so that the preference days always lie between $(c, c+1, \ldots, H)$.

\section{SCHEDULING}

The scheduling pertaining to the first day has to functions:

(1) Determination of the resource for each activity.

(2) Sequencing the activities.

Often the first function is part of the planning (i.e. loading) phase. In our case the first 
function refers only to the assignment of operators to specific operator activities, since there are no alternative machines to be selected.

In Section 3 we mentioned as our secondary objective: minimization of change-over and idle times within the time horizon. At the planning level this objective plays and essential role. Here we also want to take this objective into account. However we will use as scheduling criterion : minimize the makespan of the activities on the day list. This has two reasons. First, by minimizing the makespan one tries to obtain a schedule within the available hours per day. (If this fails the day load is too high and some machine activities should be postponed to the next day.) The second reason is that minimizing the makespan also tends to reduce the change-over times and idle times while avoiding an unbalanced loading of the machines. The constraints pertaining to the scheduling are the technological constraints and the limited number of operators, while the time window constraints are dealt with at the planning level, see Sections 4 and 5.

There are two important differences between the scheduling of this FMS and the classical job stop scheduling, which makes our problem more difficult to solve:

(a) There are sequence dependent changeover times at the turret lathe. For example, if two identical parts are processed subsequently there is a negligible change-over time, however, if these parts differ the change-over time can be considerable.

(b) There is the possibility of common use of fixtures for identical parts (for processing at the machining centre). We already indicated that loading a part onto a pallet requires two activities. The first, i.e. attaching fixtures to pallets, needs only be done once for a series of identical parts. The second one refers to clamping parts onto fixtures.

The scheduling is performed (suboptimally) in the following two steps:

Phase I: Schedulc the machining activities, assuming an unlimited number of operators.

Phase II: Schedule the operator activities, given the outcome of phase I, i.e. the machine schedules.

The reason we start with scheduling the machining activities is that the machines are the most critical resources since the machining times dominate the times needed for the other activities. After the machining sequences have been determined the tooling sequences are known. From these tool (un )loading activities can be defined. Also part transport activities can be defined. The basic idea is to group tools or parts into combined transports, given the machining sequences and the available storage capacities for either tools or parts. A more detailed description will be presented in a future paper. Phase I is solved using a branch-andbound method. The method is an adapted version of the method described by Yamamoto [5] taking into account the difference between the present scheduling problem and the classical job shop scheduling mentioned above. Phase II is essentially a project scheduling problem with limited resources, which is solved using a parallel heuristic, see [6, p. 157].

We will now discuss a simplified example to illustrate the above in more detail.

\section{Example}

In this example we consider 4 parts to be processed on the two machines. The main data are given in Table 1.

To simplify the discussion it will be assumed that part transport times can be neglected, and,

TABLE 1

Process plans

\begin{tabular}{lcll}
\hline Part & Routing & Proc. times & Pallets needed \\
\hline 1 & 2 & $8(\min )$ & P4 \\
2 & 12 & 4,7 & P1, Ps \\
3 & 12 & 6,8 & P2, P4 \\
4 & 1 & 8 & P3 \\
\hline
\end{tabular}


moreover, we do not consider fixturing (of pallets ) activities nor tool loading activities at the machines. We, furthermore, suppose that all clamping and unclamping activities take 1 minute.

At machine 1 (lathe) sequence dependent change-over times occur, caused by tool and jaw exchanges, which are given in Table 2. By a pair $(i, j)$ we denote the $j$-th operation of part $i$. Since the change-over times for machine 2 (machining centre) are not sequence dependent they are incorporated in the processing times.

From Table 1 it follows that parts 1 and 3 use the same pallet $\mathrm{P}_{4}$ for the operations $(1,1)$ and $(3,2)$, respectively. If $(1,1)$ is scheduled prior to $(3,2)$ the latter operation cannot start immediately after the former, since first part 1 must be unclamped for $\mathrm{P}_{4}$ and, subsequently, part 3 must be clamped to $\mathrm{P}_{4}$. Obviously, the same holds for the reversed order.

As a last assumption we suppose that there is one operator to perform the operator tasks.

In phase I of the scheduling the limited operator capacity is neglected. The activity network (activity on nodes) only contains machining activities. The (un) clamping times of the parts are incorporated in the network by means of transfer times between the nodes, indicated along the arrows, see Fig. 3.

Given the network in Fig. 3, one determines a minimum makespan schedule, using a branch and bound technique. For the present example the optimal sequences found are given in $\mathrm{Ta}$ ble 3 .

As a result of phase I the network in Fig. 3 is augmented with the order relations, induced by the above processing sequences, and which are

TABLE 2

Change-over times for machine 1

\begin{tabular}{llll}
\hline & $(2,1)$ & $(3,1)$ & $(4,1)$ \\
\hline$(2,1)$ & - & 2 & 4 \\
$(3,1)$ & 2 & - & 2 \\
$(4,1)$ & 4 & 2 & - \\
\hline
\end{tabular}

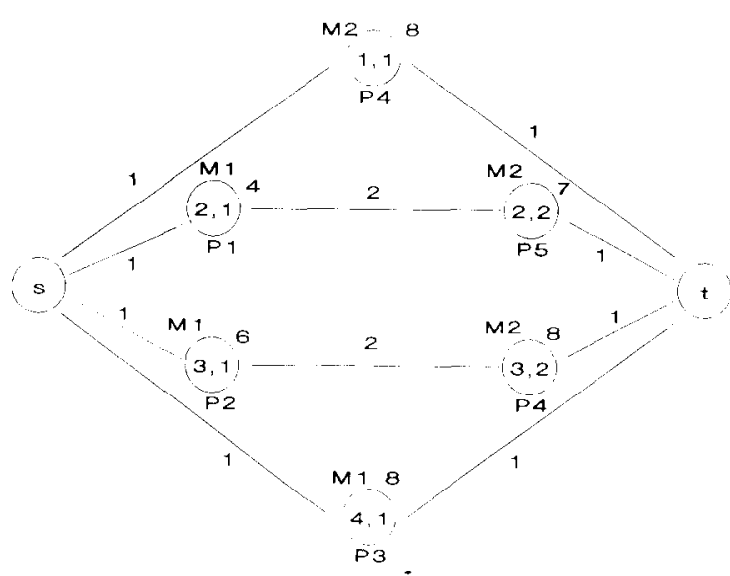

Fig. 3. Network prior to phase I.

TABIF 3

Optimal processing sequences (phase I)

\begin{tabular}{ll}
\hline Machine & Sequences \\
\hline 1 & $(2,1),(3,1),(4,1)$ \\
2 & $(1,1),(2,2),(3,2)$ \\
\hline
\end{tabular}

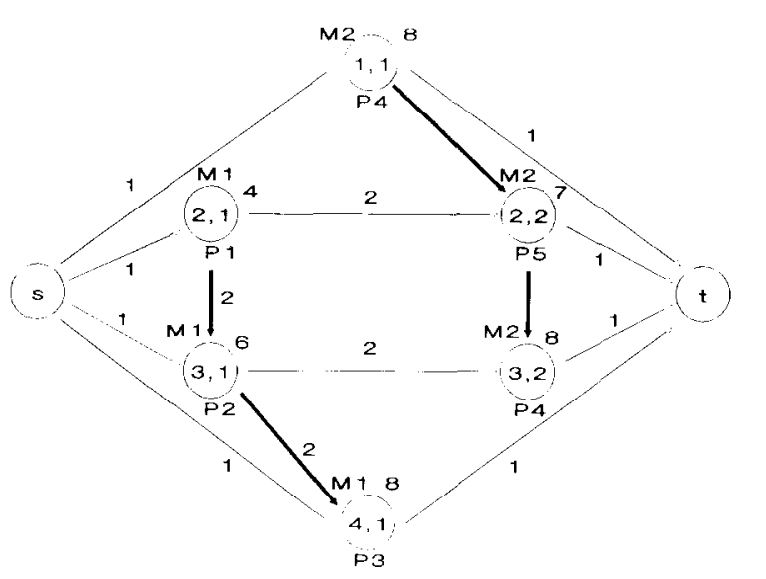

Fig. 4. Network after phase I.

denoted by the thick arrows. This network is shown in Fig. 4. The machine change-over times are indicated along these arrows.

In phase II the operator activities are scheduled, taking into account the number of available operators. The basic activity network is obtained from the network in Fig. 4 by replacing the transfer times corresponding to operator activities by separate activity nodes, 
clamping (c) and unclamping (4). This results in the network shown in Fig. 5. In this network we have omitted the durations of the activities. Note that the processing sequences will not be changes anymorc. Obscrve that the arrow from the unclamping node $(1,1)$ towards the clamping node $(3,2)$ results from the fact that the machining activity $(1,1)$ is scheduled prior to the machining activity $(3,2)$ and the fact that these parts use the same pallet $\left(\mathrm{P}_{4}\right)$.

In phase II of the scheduling the problem we now have to solve is essentially equivalent to the determination of a minimum duration project schedule with limited resources. Al-

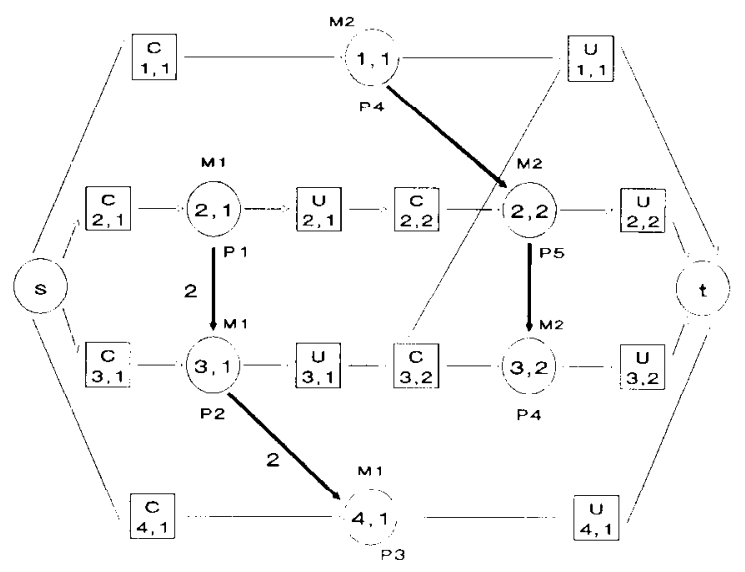

Fig. 5. Network used in phase II.

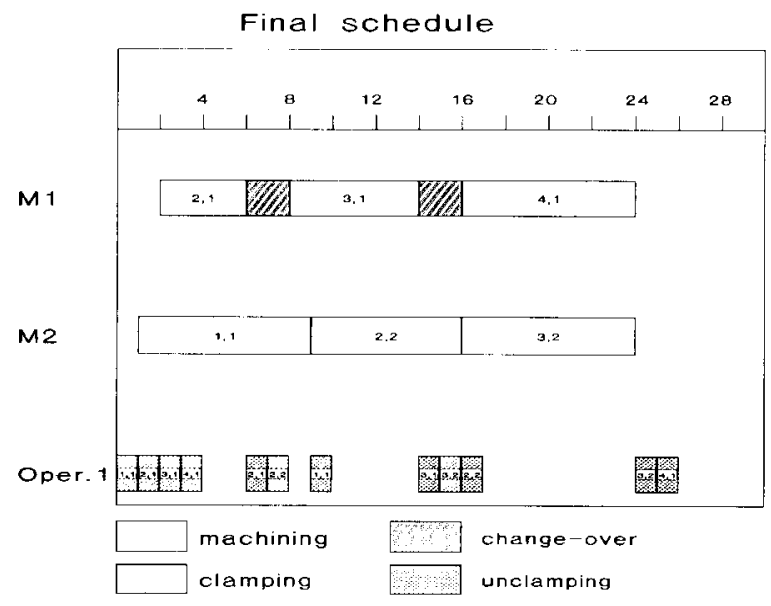

Fig. 6. Final schedule. though this problem could be solved in an optimal way, see, e.g. [6], we use an heuristic dispatching technique. If at a certain point in time two or more activities require the same opcrator, we give priority to the activity whose deferment causes the largest delay in the project duration (i.e. the makespan).

The final schedule obtained after phase II is represented in Fig. 6.

\section{CONCLUDING REMARKS}

In this paper we have described a planning/ scheduling approach for a flexible manufacturing system. The approach is developed for a specific system consisting of a turret lathe and a machining centre. The machines have a very different nature which has important implications for the planning/scheduling.

The planning/scheduling approach described in the paper has a hierarchical structure. In the planning one strives to define uniform day lists of activities taking into account the due dates. In the scheduling one schedules the activities of the first day list. The structure of the approach and some details have been discussed in the paper. In the near future we will report extensively the planning and scheduling phase.

\section{REFERENCES}

1 Hartley, J., 1984. FMS at Work. IFS (Publications) Ltd., UK.

2 Stecke, K.E., 1983. Formulation and solution of non-linear integer production planning problems for flexible manufacturing systems. Manage. Sci., 29(3): 273-288.

3 Van Looveren, A.J., Gelders, L.F. and Van Wassenhove, L.N., 1986. A review of FMS planning models. In: A. Kusiak (Ed.), From: Modelling and Design of Flexible Manufacturing Systems. Elsevier Science Publishers B.V., Amsterdam, pp. 3-31.

4 King, J.R. and Nakornchai, V., 1982. Machinecomponent group formation in group technology: review and extension. Int. J. Prod. Res., 20: 117.

5 Yamamoto, M., 1981. A program package for solving general scheduling problems. Bul. Coll. Eng., Hosei Univ., 17: 63-73.

6 Elmaghraby, S.E., 1977. Activity Networks Project Planning and Control by Network Models. Wiley, New York. 